\title{
Bandwidth, Power and Noise Considerations in Airborne cMUTs
}

\author{
Muhammed N. Senlik, Selim Olcum, H. Köymen, and Abdullah Atalar \\ Electrical and Electronics Engineering Department, Bilkent University, Ankara, TURKEY
}

\begin{abstract}
Capacitive micromachined ultrasonic transducers (cMUTs) offer wider bandwidth in air due to their low mechanical impedances. The impedance mismatch between the air and transducer decreases with the smaller device dimensions increasing the bandwidth at the expense of the degradation in the transmit power and the receive sensitivity. In this work, the bandwidth of cMUT is optimized by increasing its radiation resistance. This is done by properly choosing the size of cMUT membranes and their placement within an array. This selection not only brings an improvement in the transmitted power when it is used as a transmitter, but also improves the noise figure when it is used as a receiver. A further improvement in the noise figure is possible when the cells are clustered and connected to separate receivers.
\end{abstract}

\section{INTRODUCTION}

Performance of many airborne ultrasound applications are limited by the relatively narrow operational bandwidth. The bandwidth limitation is usually caused by the low impedance of air, resulting in poor loading of the transducer. Capacitive micromachined ultrasonic transducers (cMUTs) offer wider bandwidth in air compared to their piezoelectric alternatives.

There are various methods to further increase the bandwidth of cMUTs. For example, using thinner membranes decreases the membrane impedance and hence reduces the quality factor. Introducing lossy elements to the electrical terminals of the device may also work at the expense of reduced efficiency and sensitivity. On the other hand, increasing the radiation resistance also helps without causing a reduction in efficiency.

In this work, the performance of a cMUT array is optimized by increasing the radiation resistance of the array. This is achieved by choosing the size of cMUT membranes and their placement within the array. The proposed approach improves the bandwidth as well as the transmitted output power of the array. An increased radiation resistance also improves the noise figure of the the array when it is used as a receiver.

\section{Modelling}

A cMUT cell operates around its series resonance frequency, $f_{r}$, in the air. The displacement profile of the cMUT membrane around $f_{r}$ is [1], [2]

$$
x(r)=\sqrt{5} x_{r m s}\left(1-\frac{r^{2}}{a^{2}}\right)^{2} U(a-r)
$$

where $r$ is the radial coordinate, $a$ is the radius of the membrane and $U$ is the unit step function. $x_{r m s}$ denotes the rms displacement over the surface of the membrane [2]. The undeflected and the deflected capacitances of cMUT and its derivative with respect to $x_{r m s}$ are [1]

$$
\begin{aligned}
C_{0} & =\frac{\epsilon_{0} \pi a^{2}}{t_{g}} \\
C & =C_{0} \frac{\tanh ^{-1}\left(\sqrt{\sqrt{5} x_{r m s} / t_{g}}\right)}{\sqrt{\sqrt{5} x_{r m s} / t_{g}}} \\
\frac{d C}{d x_{r m s}} & =\frac{C_{0}}{2 x_{r m s}\left(1-\sqrt{5} x_{r m s} / t_{g}\right)}-\frac{C}{2 x_{r m s}}
\end{aligned}
$$

where $t_{g}$ is the gap height of cMUT and $\epsilon_{0}$ is the free space permittivity. The top electrode is assumed to be under the membrane surface, equivalently the membrane material is conductive. The collapse voltage, $V_{c o l}$, assuming no nonlinearity and no initial deflection under an external force is [1]

$$
V_{\text {col }}=0.39 \sqrt{\frac{16 Y_{0} t_{m}^{3} t_{g}^{3}}{\left(1-\nu^{2}\right) \epsilon_{0} a^{4}}}
$$

where $t_{m}$ is the thickness of the membrane and $Y_{0}$ and $\nu$ are the Young's modulus and the Poisson's ratio of the membrane material, respectively. If the operating voltage is $V_{D C}$ then the turns ratio, $n$, in the Mason's equivalent circuit [3], Fig. 1 is [1]

$$
n=V_{D C} \frac{d C}{d x_{r m s}}
$$

The mechanical impedance of the membrane around $f_{r}$ can

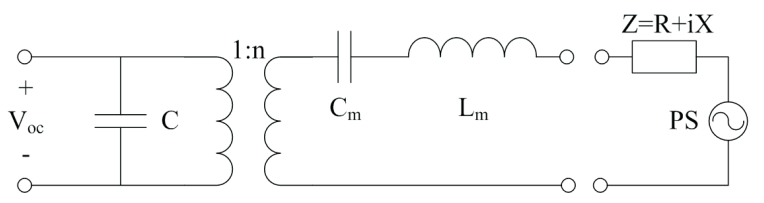

Fig. 1. The Mason's equivalent circuit of cMUT [3]. $C$ is the shunt input capacitance and $n$ is the turns ratio. The membrane impedance around $f_{r}$ is modelled with a series LC section [1]. $Z=R+i X$ is the radiation impedance of the membrane. During the reception, cMUT is excited by a force source with an amplitude of $P S$, where $P$ is the incident pressure field and $S$ is the area of a single cMUT membrane.

be modelled with a series LC section, whose values are [1]

$$
\begin{aligned}
L_{m} & =\pi a^{2} t_{m} \rho \\
C_{m} & =\frac{\left(1-\nu^{2}\right) a^{2}}{8.9 \pi Y_{0} t_{m}^{3}}
\end{aligned}
$$


where $\rho$ is the density of the membrane material. The radiation impedance of the cMUT cell in an array is [2]

$$
Z_{r}=R_{r}+i X_{r}
$$

Since the acoustic loading is low, the effect of the radiation reactance is ignored.

A circular array, where the cells are placed in a hexagonal pattern, as depicted in Fig. 2 is investigated. The center-tocenter spacing between the cells is $d$. The effective radius of the array, $r$, is equal to $a \sqrt{N / f_{F}}$, where $N$ is the number of the cells and $f_{F}=(2 \pi / \sqrt{3})(a / d)^{2}$ is the fill factor.

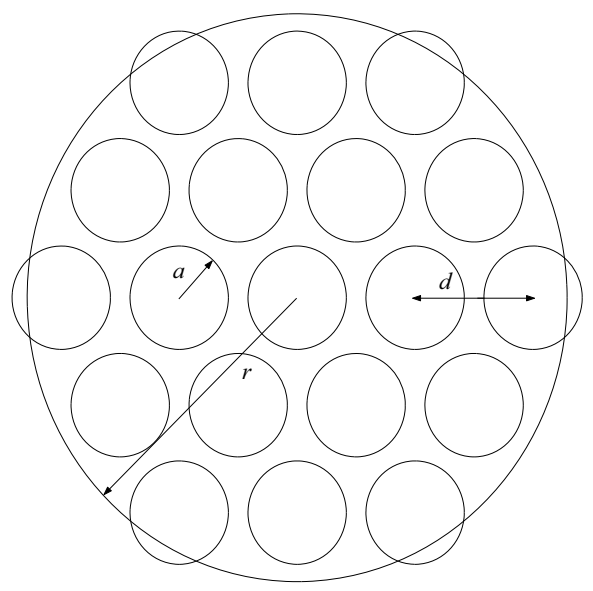

Fig. 2. The geometry of a circular array with hexagonally placed $N=19$ cells. $a$ and $d$ are the radius of the cell and the center-to-center spacing between the cells, respectively. $r$ is the effective radius of the array.

\section{Performance Figures}

In this section, the effects of the cell dimensions and the center-to-center spacing between the cells on $Q$ factor and transmit and receive performances are investigated. The membrane material is assumed to be silicon, whose properties can be found in Table I. The operating frequency and the collapse voltage are chosen to be $100 \mathrm{kHz}$ and $500 \mathrm{~V}$, respectively. Throughout this section, the effective radius of the array $(r)$ is kept constant at $10 \mathrm{~mm}$ to be able to make a fair comparison. The radius of a single membrane $(a)$ is varied between $1 \mathrm{~mm}$ and $2 \mathrm{~mm}$. As $a$ is changed, the thickness of the membrane and the gap height is adjusted to meet the center frequency and collapse voltage specifications. The effect of the atmospheric deflection is taken into account in the calculation of $t_{g}$ [1]. The dimensions of cMUTs used in the simulations can be found in Table II. $d$ is varied between $2 a$ and $3 a$. In order to keep $r$ constant, the number of cells in the array $(N)$ is adjusted as an integer variable.

\section{A. Radiation Resistance}

In [2], it is shown that the radiation resistance of a cMUT cell in an array is a strong function of separation between the cells $(d)$. It is maximized, when $d$ is around $1.25 \lambda$ for the most
TABLE I

Material Parameters Used In The Simulations.

\begin{tabular}{lll}
\hline Parameter & $\mathrm{Si}$ & Air \\
\hline Young's modulus $(\mathrm{GPa})$ & 169 & \\
Poisson's ratio & 0.27 & \\
Density $\left(\mathrm{kg} / \mathrm{m}^{3}\right)$ & 2332 & 1.27 \\
Speed of sound $(\mathrm{m} / \mathrm{s})$ & & 331 \\
Atmospheric pressure $(\mathrm{kPa})$ & & 101 \\
\hline
\end{tabular}

TABLE II

Device Dimensions Used In The Simulations.

\begin{tabular}{cccc}
\hline $\begin{array}{c}a \\
(\mathrm{~mm})\end{array}$ & $\begin{array}{c}t_{m} \\
(\mu \mathrm{m})\end{array}$ & $\begin{array}{c}t_{g} \\
(\mu \mathrm{m})\end{array}$ & $\begin{array}{c}t_{g}^{\prime} \\
(\mu \mathrm{m})\end{array}$ \\
\hline 1 & 24 & 13 & 0.87 \\
1.25 & 37 & 10 & 0.79 \\
1.5 & 54 & 8 & 0.72 \\
1.75 & 73 & 6.8 & 0.67 \\
2 & 95 & 6 & 0.63 \\
\hline
\end{tabular}

compact arrangement $(d=2 a)$. Such an arrangement requires relatively large radius cells with relatively thick membranes. A smaller cell radius would allow a thinner membrane with a potentially better bandwidth. In order to increase the radiation resistance for a smaller cell size, $d$ is made larger than $2 a$ to get a sparse arrangement of the cells. Fig. 3 shows $R_{r}$ of a single cell in an array made of different cMUTs as a function of $d / a$. As depicted in Fig. 3, $R_{r}$ is maximized at a lower $a$ value as $d / a$ is increased. At these points, the loading on each cMUT is maximized [2], [4]. Note that, for a membrane with $a=1 \mathrm{~mm}, R_{r}$ is more than three times higher when $d / a=2.8$ compared to the most compact arrangement of $d / a=2$.

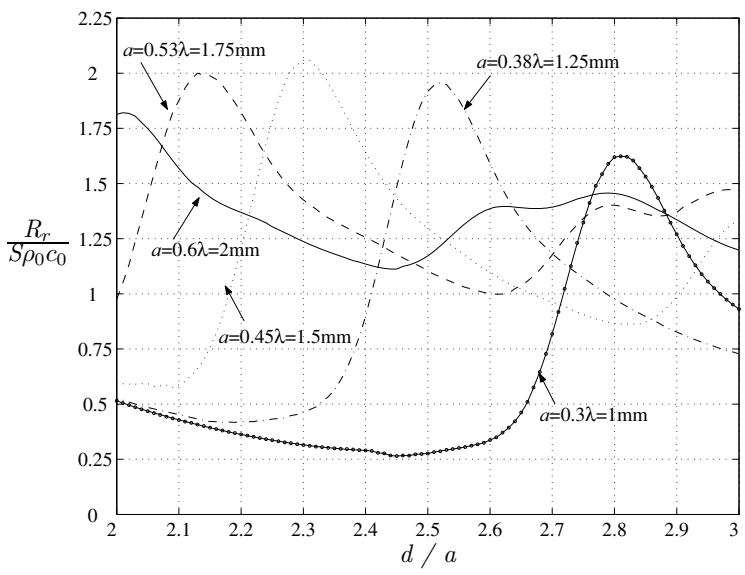

Fig. 3. The radiation resistance, $R_{r}$, normalized by $S \rho_{0} c_{0}$ of a single cell in various arrays as a function of $d / a$.

\section{B. $Q$ Factor}

In the air, $Q$ is determined by the series RLC section at the mechanical side of the Mason's equivalent circuit [5]. Hence

$$
Q=\frac{2 \pi f_{r} L_{m}}{R_{r}}
$$

Using (5) $Q$ can be written in the form

$$
Q=\frac{2 \pi f_{r} \pi a^{2} t_{m} \rho}{R_{r}}
$$


As seen from this equation, a smaller cell size, $a$, helps reduce $Q$. Moreover, a small $a$ also requires in a thinner membrane, $t_{m}$ to keep $f_{r}$ constant. Fig. 4 shows $Q$ of cMUT array as a function of $d / a$. In the most compact arrangement, $Q$ of the membrane with $a=2 \mathrm{~mm}$ is lower compared to the ones with $a=1.5 \mathrm{~mm}$ and $1.75 \mathrm{~mm}$ due to a higher $R_{r}$ value. As $d$ increases, $Q$ of each array has a minimum at the point, when $R_{r}$ is maximized. For the most compact arrangement, $Q$ for all devices are above 150 , however with a sparse arrangement, it is possible to obtain $Q$ around 50 without introducing any lossy elements to the system.

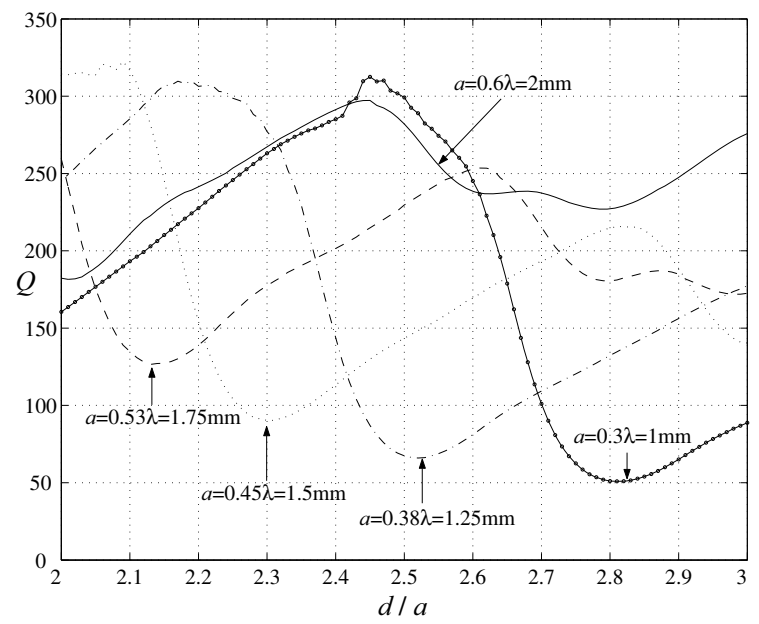

Fig. 4. $\quad Q$ of cMUT array as a function of $d / a$.

\section{Transmit Mode}

To maximize the power transferred to the medium, cMUT is driven such that the membrane swings the entire stable gap height, $t_{g}^{\prime}$ (the allowed swing range of the membrane without ignoring the atmospheric deflection). The velocity of the membrane will be sinusoidal with frequency $f_{r}$, since $Q$ is relatively high. Then, the rms velocity of the membrane in the time domain is

$$
v_{r m s}=\frac{2 \pi f_{r} x_{r m s}}{\sqrt{2}}=\frac{2 \pi f_{r} t_{g}^{\prime}}{2 \sqrt{2}}
$$

and the average output power from the array is

$$
P_{\text {ave }}=N v_{r m s}^{2} R_{r}
$$

Fig. 5 shows $P_{\text {ave }}$ of various arrays as a function of $d / a$. $P_{\text {ave }}$ is maximized, when $R_{r}$ is maximized. For $a=1.5 \mathrm{~mm}$ and $1.75 \mathrm{~mm}$, it is possible to obtain $9 \mathrm{~mW}$ power from the array. Note that as $d / a$ increases, $N$ decreases. Consequently, $P_{\text {ave }}$ is only 1.5 times higher, although the increase in $R_{r}$ can be more than 3 times compared to the most compact arrangement.

\section{Receive Mode}

The receive performance of a transducer is specified by its open circuit-voltage, $V_{o c}$. The Mason's equivalent circuit in Fig. 1 is used to calculate $V_{o c}$. cMUT is excited by a force

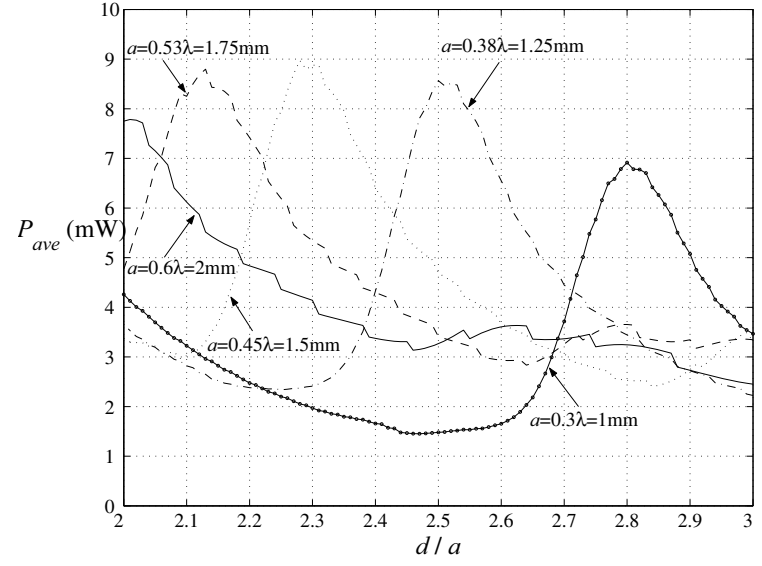

Fig. 5. The average output power of various arrays as a function of $d$.

source with an amplitude of $P S$ where $P$ is the incident pressure field and $S$ is the single cell area. The ratio of the operating voltage to the collapse voltage, $V_{D C} / V_{c o l}$, is assumed to be 0.9 . It is found that $V_{o c}$ is nearly independent of $R_{r}$ and is calculated as $10.5,7.5,5.7,4.6$ and $3.8 \mathrm{~V} / \mathrm{Pa}$ as $a$ is increased from $1 \mathrm{~mm}$ to $2 \mathrm{~mm}$ in $0.25 \mathrm{~mm}$ steps. Using Fig. 1, $V_{o c}$ at the resonance is written as

$$
V_{o c}=\frac{P S}{n}
$$

since the shunt input capacitance, $C$, shows a high impedance compared to the rest of the network and can be ignored. It can be shown that $n$ gets smaller for a smaller $a$, and hence $V_{o c}$ increases for smaller cells.

\section{E. Noise Analysis}

An important figure of merit for the receive performance is the noise figure, $F$. In the receive circuitry, a very low noise OPAMP, MAXIM MAX410, in a non-inverting configuration is used as shown in Fig. 6(a). The input noise voltage, $e_{n}$, and noise current, $i_{n}$, of this OPAMP are $1.2 \mathrm{nV} / \mathrm{Hz}^{1 / 2}$ and $1.2 \mathrm{pA} / \mathrm{Hz}^{1 / 2}$, respectively. The feedback resistors, $R_{1}$ and $R_{2}$, are chosen as $1 \mathrm{k} \Omega$ and $10 \mathrm{k} \Omega$ to obtain a gain of 11 . The noise contributions from the resistors can be decreased by connecting parallel capacitors, $C_{1}$ and $C_{2}$, with values of $100 \mathrm{nF}$ and $10 \mathrm{nF}$. At $100 \mathrm{kHz}$, the optimum source impedance to minimize the noise figure, $Z_{\text {opt }}$, is $(1250+\mathrm{j} 14) \Omega$ giving $F_{\text {min }}=0.9 \mathrm{~dB}$. (For the inverting configuration of the same OPAMP, $Z_{\text {opt }}=29+j 170 \Omega$ giving $F_{\min }=1.4 \mathrm{~dB}$.)

Fig. 6(b) shows $F$ of various cell sizes as a function of $d / a$. The noise figure shown also includes the negative effect of the fill factor as $d / a$ is increased. A high $R_{r}$ at a high $d / a$ value may not result in a low noise figure due to a low fill factor at that point. It is found that clustering a number of cells to separate OPAMPs as shown in Fig. 7(a) will result in even lower noise figure. Fig. 7(b) shows the optimum number of cells per cluster and the number of clusters as a function of $d / a$ for an airborne cMUT array with $a=1 \mathrm{~mm}$. The resulting $F$ is shown in Fig. 6(b). It is possible to approach $F_{\text {min }}$ of 
the OPAMP by using 8 OPAMPs each connected to 12 cells with $d / a=2$.

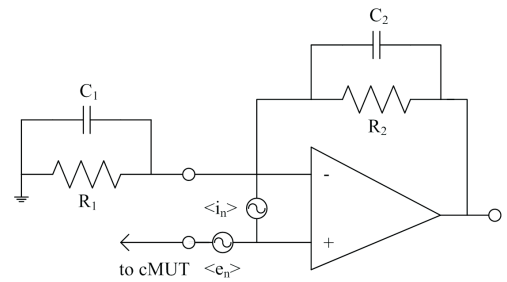

(a)

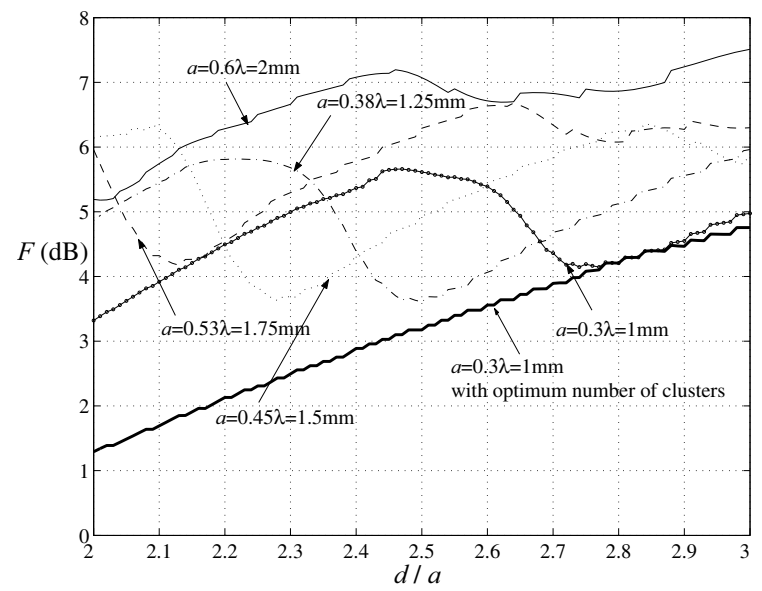

(b)

Fig. 6. (a) The receiver circuitry used in the calculation of the noise figure (b) Noise figure of various arrays as a function of $d / a$. Upper curves show the noise figure when all cMUT cells are connected to a single OPAMP. The lower curve is the minimized noise figure for $a=1 \mathrm{~mm}$ when an optimum number of cells are connected to separate OPMAPs.

\section{CONCLUSIONS}

The bandwidth of cMUT in air is wider compared to their piezoelectric alternatives. In this work, it is shown that by properly choosing the cell size and the distance between the cells, it is possible to optimize the radiation resistance of cMUT. This brings improvement in bandwidth and transmit power. It is shown that open circuit voltage of cMUT in air is nearly independent of radiation resistance.

A state of the art low noise OPAMP is used in the receiver circuitry to calculate the noise figure of cMUT. It is found that noise figure can be minimized by properly adjusting the number of cells connected to the receiver. It is shown that a noise figure approaching the minimum noise figure of the receiver is possible if the cells are clustered and connected to separate receivers.

\section{ACKNOWLEDGEMENT}

This work is supported in part by Turkish Scientific and

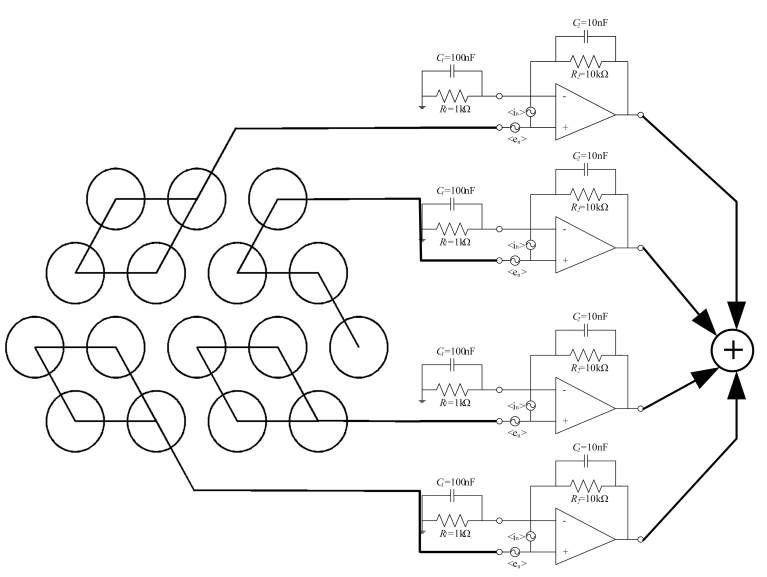

(a)

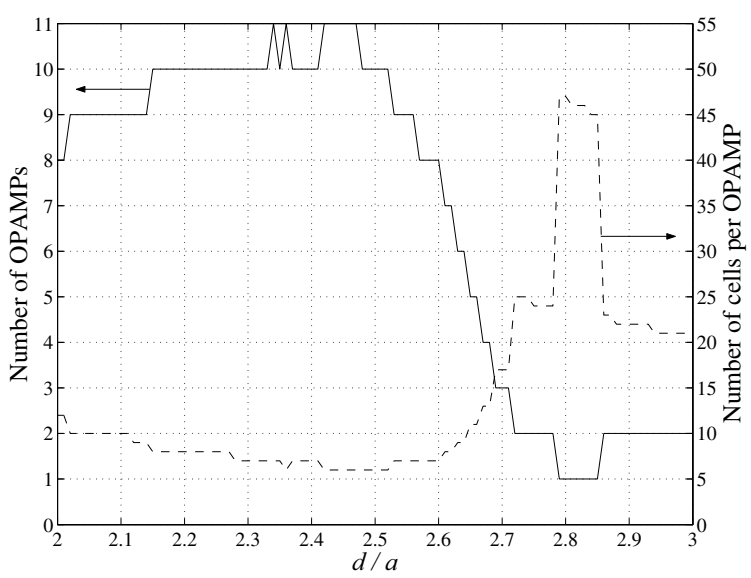

(b)

Fig. 7. (a) Method of connecting clusters to separate OPAMPs. (b) Optimum number of cluster size and number of OPAMPs to minimize the noise figure as a function of $d / a$ for $a=1 \mathrm{~mm}$.

Research Council (TUBITAK) under project grants 105E23 and 107T921. SO acknowledges the support of TUBITAK and ASELSAN for their Ph.D. Scholarship Programs. AA thanks TUBA for the research support.

\section{REFERENCES}

[1] I. O. Wygant, M. Kupnik, and B. T. Khuri-Yakub, "Analytically calculating membrane displacement and the equivalent circuit model of a circular cMUT cell," in Proc. IEEE Ultrason. Symp., 2008, 2111-2114.

[2] M. N. Senlik, S. Olcum, H. Koymen, and A. Atalar, "Radiation impedance of an array of circular capacitive micromachined ultrasonic transducers," IEEE Trans. Ultrason., Ferroelect., Freq. Contr., submitted for publication.

[3] M. I. Haller and B. T. Khuri-Yakub, "A surface micromachined electrostatic ultrasonic air transducer," IEEE Trans. Ultrason., Ferroelect., Freq. Contr., vol. 43, pp. 1-6, 1996.

[4] H. Lee, J. Tak, W. Moon, and G. Lim, "Effects of mutual impedance on the radiation characteristics of transducer arrays," J. Acoust. Soc. Am., vol. 115, pp. 666-679, 2004.

[5] S. Olcum, A. Atalar, H. Köymen, and M. N. Senlik, "Stagger tuned cMUT array for wideband airborne applications," in Proc. IEEE Ultrason. Symp., 2006, pp. 2377-2380. 